\title{
Three-dimensional rotational averages in radiation-molecule interactions: an irreducible cartesian tensor formulation
}

\author{
David L Andrews and Nick P Blake \\ School of Chemical Sciences, University of East Anglia, Norwich NR4 7TJ, UK
}

Received 23 May 1988, in final form 18 July 1988

\begin{abstract}
In this paper we present a new method for the calculation of the rotational averages which arise in the theory of spectroscopic radiation-molecule interactions in fluid media. Based upon the principles of irreducible cartesian tensor analysis, the method presented allows us to express results either in the usual reducible form, or directly in terms of linearly independent sets of irreducible tensor products. For interactions up to and including rank 3 in the molecular response (or non-linear susceptibility) tensor, the rotational averages cast in terms of irreducible tensor products are considerably simpler in structure than the corresponding results expressed in reducible form.
\end{abstract}

\section{Introduction}

In the study of molecule-radiation interactions it is often the case that the principal observable, for example the quantum transition rate or scattering intensity, depends upon the orientation of sample molecules with respect to the radiation field. Within the framework of molecular quantum electrodynamics, the rate and intensity parameters associated with such interactions are normally described in terms of the Fermi golden rule. Most processes are incoherent, in the sense that they do not depend on exact phase-matching between the incident and any emergent photons, and the observable can be expressed in terms of the square modulus of a quantum-mechanical probability amplitude, $M_{f i}$, constructed from a contraction of two tensors. Adopting the implied summation convention for repeated tensor indices, we can express $M_{f i}$ for a process which involves $n$ photon-molecule interactions occurring at any one centre as a series of the form

$$
M_{f i}=S_{i_{1} \ldots i_{n}} T_{i_{1} \ldots i_{n}}+S_{i_{1} \ldots i_{n+1}}^{\prime} T_{i_{1} \ldots i_{n+1}}^{\prime}+\ldots
$$

Here the first term, comprising the contraction of two rank- $n$ tensors, represents the leading term, usually the electric dipole approximation, for the $n$ photon-molecule interaction, whilst successive terms involving higher-rank tensor contractions represent higher-order multipolar corrections to the probability amplitude: the tensors $T_{i_{1} \ldots i_{n}}$ and $T_{i_{1} \ldots i_{n+1}}^{\prime}$ are the corresponding molecular response tensors, and $S_{i_{1} \ldots i_{n}}$ and $S_{i_{1} \ldots i_{n+1}}^{\prime}$ are the polarisation tensors constructed from products of radiation field vectors [1].

Taking the square modulus of the matrix element gives

$$
\begin{gathered}
\left|M_{j i}\right|^{2}=S_{i_{1} \ldots i_{n}} \bar{S}_{j_{1} \ldots j_{n}} T_{i_{1} \ldots i_{n}} \bar{T}_{j_{1} \ldots j_{n}}+S_{i_{1} \ldots i_{n}} \bar{S}_{j_{1} \ldots j_{n+1}}^{\prime} T_{i_{1} \ldots i_{n}} \bar{T}_{j_{1} \ldots j_{n+1}}^{\prime} \\
+\bar{S}_{i_{1} \ldots i_{n}} \bar{S}_{j_{1} \ldots j_{n+1}}^{\prime} \bar{T}_{i_{1} \ldots i_{n}} T_{j_{1} \ldots j_{n+1}}^{\prime}+\ldots
\end{gathered}
$$


If the sample is a fluid or gas it is necessary to account for the random orientation of the molecules within the sample. To this end it is usually necessary to rotationally average a result derived for the case where the laboratory and molecular frames are fixed with respect to one another. For such systems, the leading term for the observable, $A^{(2 n)}$, can be written as

$$
A^{(2 n)}=\left\langle T_{i_{1} \ldots i_{n}} S_{i_{1} \ldots i_{n}} \bar{T}_{i_{n+1} \ldots i_{2 n}} \bar{S}_{i_{n+1} \ldots i_{2 n}}\right\rangle
$$

where the angle brackets denote the rotational average. Thus, for example, singlephoton absorption $(n=1)$ requires a rank-2 tensor average, two-photon absorption and Raman scattering $(n=2)$ require a fourth-rank rotational average, and three-photon absorption and hyper-Raman scattering $(n=3)$ require elucidation of the sixth-rank rotational average [2-9].

In the normal trigonometric method for deriving the appropriate averages, the molecular-fixed parameters are first transformed into a molecular-fixed frame, denoted in this paper by Greek indices, through the relation

$$
T_{i_{1} \ldots i_{n}}=l_{i_{1} \lambda_{1}} \ldots l_{i_{n} \lambda_{n}} T_{\lambda_{1} \ldots \lambda_{n}}
$$

where $l_{\lambda_{j} i_{P}}$ represents the direction cosine of the angle between the molecule-fixed $\lambda_{j}$ axis and the laboratory-fixed $i_{P}$ axis, as may be expressed in terms of Euler angles $\varphi$, $\theta$ and $\psi$. The required even-rank rotational average is then obtained by inserting equation (1.4) into (1.3) and then integrating over the Euler angles, i.e.

$$
\begin{aligned}
A^{(2 n)}=\left(8 \pi^{2}\right)^{-1} & T_{i_{1} \ldots i_{n}} \bar{T}_{i_{n+1} \ldots i_{2 n}} S_{\lambda_{1} \ldots \lambda_{n}} \bar{S}_{\lambda_{n+1} \ldots \lambda_{2 n} n} \\
& \times \int_{0}^{2 \pi} \int_{0}^{\pi} \int_{0}^{2 \pi} l_{i_{1} \lambda_{1}} \ldots l_{i_{2 n} \lambda_{2 n}} \sin \theta \mathrm{d} \varphi \mathrm{d} \theta \mathrm{d} \psi .
\end{aligned}
$$

As $n$ increases, the trigonometric evaluation of results of this form becomes a problem of rapidly escalating difficulty. Each index can represent either $x, y$ or $z$, and hence there are $3^{4 n}$ separate integrals to evaluate. The problem of isotropically averaging tensor quantities of this type has often been addressed in the limited context of particular physical processes. In earlier papers, Andrews et al $[7,10]$ developed a systematic, non-trigonometric, matrix-based procedure, giving explicit results up to rank $8(n=4)$ whilst the logistics of the problem recently led Wagnière [8] and McClain et al [9] to evaluate the integrals computationally, obtaining results up to rank $10(n=5)$.

In $\$ 2$ of this paper it is shown that, in the non-trigonometric method of rotational averaging, expressions of the form of equation (1.3) can be further simplified by casting both the molecular and polarisation tensors into their embedded irreducible cartesian forms. This method has two distinct advantages. First, the results are cast in terms of a linearly independent irreducible basis set in which each component can be characterised by its rotational transformation properties: previous averaging treatments have generally necessitated subsequent irreducible tensor development in order to perform a full symmetry analysis. Second, the transformation to an irreducible basis set means that the rotational averaging matrices are brought into block diagonal form, simplifying the results considerably. In $\$ 3$, development of these results illustrates their correspondence with conventional rotational averages, while in $\S 4$ it is demonstrated that the procedure can be extended to tensor averages of odd rank, so facilitating evaluation of the rotational averages of the higher-order correction terms in equation (1.2). 


\section{Calculational procedure}

The procedure for calculation of the rotational averages of the type given in equation (1.3) involves consideration of the linear transformation properties of the tensors involved. Since the integrand in (1.5) must be rotationally invariant, it forms a basis for a totally symmetric irreducible representation of the rotation-inversion group $\mathrm{SO}(3)$. We can ascertain the exact form of this basis by expressing each tensor as a sum of its embedded irreducible tensor components [11-13]†.

In general, a reducible tensor of rank $n$ can be written as a sum of irreducible parts of weights $j(0 \leqslant j \leqslant n)$, each of which is associated with a multiplicity $N_{n}^{(j)}$ given by [14]

$$
N_{n}^{(j)}=\sum_{k}(-1)^{k}\left[\begin{array}{l}
n \\
k
\end{array}\right]\left[\begin{array}{c}
2 n-3 k-j-2 \\
n-2
\end{array}\right]
$$

where $0 \leqslant k \leqslant[(n-j) / 3]$. Each irreducible tensor has $(2 j+1)$ independent components, so that the total number of components is

$$
\sum_{j}(2 j+1) N_{n}^{(j)}=3^{n}
$$

as required. The reduction of the tensors in (1.2) into irreducible parts thus takes the following form:

$$
\begin{aligned}
& T_{i_{1} \ldots i_{n}}=\sum_{j=0}^{n} \sum_{p=1}^{N_{n}^{(j)}} T_{i_{1} \ldots i_{n}}^{(j ; p)} \\
& \bar{T}_{i_{n+1} \ldots i_{2 n}}=\sum_{j^{\prime}=0}^{n} \sum_{q=1}^{N_{n}^{\left(j^{\prime}\right)}} \bar{T}_{i_{n+1} \ldots i_{2 n}}^{\left(j^{\prime} ; q\right)} \\
& S_{i_{1} \ldots i_{n}}=\sum_{j^{\prime \prime}=0}^{n} \sum_{r=1}^{N_{n}^{\left(j^{\prime \prime}\right)}} S_{i_{1} \ldots i_{n}}^{\left(j^{\prime \prime} ; r\right)} \\
& \bar{S}_{i_{n+1} \ldots i_{2 n}}=\sum_{j^{\prime \prime \prime}=0}^{n} \sum_{s=1}^{N_{n}^{\left(j^{\prime \prime \prime}\right)}} \bar{S}_{i_{n+1}\left(j^{\prime \prime \prime} ; s\right)} .
\end{aligned}
$$

We now consider the substitution of these results into (1.2), where there are index contractions involving coupling between the tensors represented by (2.3) and (2.5), and also between those represented by (2.4) and (2.6). It is well known that the coupling of two tensors of weights $j$ and $j^{\prime \prime}$ results in a tensor represented by weights $j^{*}$ in the range $\left|j-j^{\prime \prime}\right| \leqslant j^{*} \leqslant j+j^{\prime \prime}$. However, the $n$-fold contraction of two tensors of rank $n$ must give rise to a scalar result; therefore products of the type $T_{i_{1} \ldots i_{n}}^{(j ; p)} S_{i_{1} \ldots i_{n}}^{\left(j^{\prime \prime} ; r\right)}$ will only be non-vanishing when $j^{*}=0$, and this can only occur when $j=j^{\prime \prime}$. Similar reasoning shows that $j^{\prime}=j^{\prime \prime \prime}$, and hence the substitution of equations (2.3)-(2.6) into (1.2), must give the result

$$
\left\langle\left|T_{i_{1} \ldots i_{n}} S_{i_{1} \ldots i_{n}}\right|^{2}\right\rangle=\sum_{j, j^{\prime}=0}^{n} \sum_{p, q}^{N_{n}^{(j)}} \sum_{r, s}^{N_{n}^{\left(j^{\prime}\right)}}\left\langle T_{i_{1} \ldots i_{n}}^{(j ; p)} \bar{T}_{i_{n+1} \ldots i_{2 n}}^{\left(j^{\prime} ; q\right)} S_{i_{i 1} \ldots i_{n}}^{(j ; r)} \bar{S}_{i_{n+1} \ldots i_{2 n}}^{\left(j^{\prime} ; s\right)}\right\rangle .
$$

Equation (2.7) can be re-expressed in a natural form $[12,13]$ through the use of the

$\uparrow$ Note that in [12] the last two column headings of table III should be interchanged, and $T_{(j \mid n)}$ becomes $T_{(n \mid j)}$. 
mapping formulae

$$
\begin{aligned}
& T_{i_{1} \ldots i_{n}}^{(j ; p)}=G_{i_{1} \ldots i_{n} ; k_{1} \ldots k_{j}}^{(0 ; p)} t_{k_{1} \ldots k_{j}}^{(j ; p)} \\
& \bar{T}_{i_{n+1} \ldots i_{2 n}}^{\left(j^{\prime} ; q\right)}=G_{i_{n+1} \ldots i_{2 n} ; l_{i} \ldots l_{j^{\prime}}}^{(0 ; q)} \bar{t}_{l_{1} \ldots l_{j^{\prime}}}^{\left(j^{\prime} ; q\right)} \\
& S_{i_{1} \ldots i_{n}}^{(j ; r)}=G_{i_{1} \ldots i_{n} ; m_{1} \ldots m_{j}}^{(0 ; r)} S_{m_{1} \ldots m_{j}}^{(j ; r)} \\
& \bar{S}_{i_{n+1} \ldots i_{2 n}}^{\left(j^{\prime} ; s\right)}=G_{i_{n+1} \ldots i_{2 n} ; n_{1} \ldots n_{j^{\prime}}}^{(0 ; s)} \bar{s}_{n_{1} \ldots n_{j^{\prime}}}^{\left(j^{\prime} ; s\right)} .
\end{aligned}
$$

Here $G_{i_{1} \ldots i_{n} ; n_{1} \ldots n_{j}}^{(0 ; s)}$ represents a rank-(n+j)-invariant symmetry preserving mapping from the weight $-j$, rank- $j$ subspace into a weight $-j$, rank- $n$ irreducible subspace. Substitution of these identities into $(2.7)$ gives

$$
\begin{aligned}
& \left\langle\left|T_{i_{1} \ldots i_{n}} S_{i_{1} \ldots i_{n}}\right|^{2}\right\rangle
\end{aligned}
$$

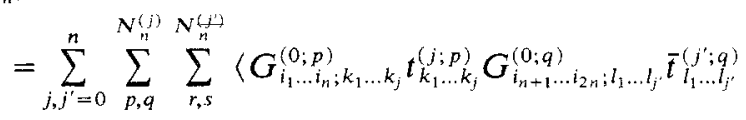

$$
\begin{aligned}
& \left.\times G_{i_{1} \ldots i_{n} ; m_{1} \ldots m_{j}}^{(0 ; r)} s_{m_{1} \ldots m_{j}}^{(j ; r)} G_{i_{n+1} \ldots i_{2 n} ; n_{1} \ldots n_{j^{\prime}}}^{(0 ; s)} \bar{s}_{n_{1} \ldots n_{j}}^{\left(j^{\prime} ; s\right)}\right\rangle .
\end{aligned}
$$

In order to develop (2.12) further, we note that by projecting the two invariant mappings given expression in (2.8) and (2.9) to their dual bases, through use of the metric tensor $g_{p q^{\prime}}^{(n ; j)}$, i.e.

$$
\begin{aligned}
& G_{i_{1} \ldots i_{n} ; k_{1} \ldots k_{j}}^{(0 ; ;)}=\sum_{p^{\prime}} g_{p p^{\prime}}^{(n ; j)} \tilde{G}_{k_{1} \ldots k_{j} ; i_{1} \ldots i_{n}}^{\left(0 ; p^{\prime}\right)} \\
& G_{i_{n+1}, \ldots, q, i_{2 n} ; l_{1} \ldots l_{j^{\prime}}}^{(0 ; q)}=\sum_{q^{\prime}} g_{q q^{\prime}}^{\left(n ; j^{\prime}\right)} \tilde{G}_{l_{1} \ldots j_{j^{\prime}} ; i_{n+1} \ldots i_{2 n}}^{\left(0 ; j^{\prime}\right)}
\end{aligned}
$$

it is possible to conduct an $n$-fold index contraction to give a new mapping between two rank- $j$ subspaces $[12,13]$ :

$$
\tilde{G}_{k_{1} \ldots k_{j} ; i_{1} \ldots i_{n}}^{\left(0 ; p^{\prime}\right)} G_{i_{1} \ldots i_{n} ; m_{1} \ldots m_{j}}^{(0 ; r)}=\delta_{p^{\prime} r} E_{k_{1} \ldots k_{j} ; m_{1} \ldots m_{j}}^{(j)} .
$$

The invariant tensor mapping operator $E_{k_{1} \ldots k_{j} ; m_{1} \ldots m_{j}}^{(j)}$ represents a mapping from the rank- $j$ space to the natural rank- $j$, and hence weight- $j$, subspace. By exploiting the idempotent nature of this operator when it operates upon rank- $j$ natural tensors, substitution of equations (2.13)-(2.15) into (2.12) gives the result

$$
\left\langle\left|T_{i_{1} \ldots i_{n}} S_{i_{1} \ldots j_{n}}\right|^{2}\right\rangle=\sum_{j=0}^{n} \sum_{p, q}^{N_{n}^{(j)}} \sum_{r, s}^{N_{n}^{\left(j^{\prime}\right)}} g_{p r}^{(n ; j)} g_{q s}^{(n ; j)}\left\langle t_{k_{1} \ldots k_{j}}^{(j ; p)} \bar{t}_{l_{1} \ldots j_{j}}^{\left(j^{\prime} ; q\right)} s_{k_{1} \ldots k_{j}}^{(j ; r)} \bar{s}_{l_{1} \ldots l_{j}}^{\left(j^{\prime} ; s\right)}\right\rangle .
$$

The isotropic average can now be directly evaluated, by taking the weight- 0 isotropic part of the tensor product $t_{k_{1} \ldots k_{j}}^{(j ; p)} \bar{t}_{l_{1} \ldots j_{j^{\prime}}}^{\left(j^{\prime} ; q\right)}$ and contracting the result with $s_{k_{1} \ldots k_{j}} \bar{s}_{l_{1} \ldots l^{\prime}}$. As before, the isotropic part of such tensor products is only non-vanishing when $j=j^{\prime}$, and the relation takes the form

$$
\left(t_{k_{1} \ldots k_{j}}^{(j ; p)} t_{l_{1} \ldots l_{j}}^{\left(j^{\prime} ; q\right)}\right)^{(0)}=\delta_{j j^{\prime}} t_{i_{1} \ldots i_{j}}^{(j ; p)} t_{i_{1} \ldots i_{j}}^{(j ; q)}(2 j+1)^{-1} E_{k_{1} \ldots k_{j} ; l_{1} \ldots l_{j}}^{(j)}
$$

which on substitution into $(2.16)$ gives

$$
\begin{aligned}
& \left\langle\left|T_{i_{1} \ldots i_{n}} S_{i_{1} \ldots i_{n}}\right|^{2}\right\rangle \\
& =\sum_{j=0}^{n}(2 j+1)^{-1} \sum_{p, q}^{N_{n}^{(j)}} \sum_{r, s}^{N_{h}^{\left(h^{\prime}\right)}} g_{p r}^{(n ; j)} g_{q s}^{(n ; j)} t_{\lambda_{1} \ldots \lambda_{j}}^{(j ; p)} \bar{t}_{\lambda_{1} \ldots \lambda_{j}}^{(j ; q)} s_{k_{1} \ldots k_{j}}^{(j ; r)} \bar{s}_{k_{1} \ldots k_{j}}^{(j ; s)} .
\end{aligned}
$$

This is the central result for the rotational average, as expressed in terms of natural tensors. It is important to note that it is only those tensor products where all weights are the same that contribute to the average. 
Equation (2.18) can be suitably embedded in the computationally more convenient rank- $n$ tensor space by use of the following identity proved in appendix 1:

$t_{\lambda_{1} \ldots \lambda_{j}}^{(j ; p)} t_{\lambda_{1} \ldots \lambda_{j}}^{(j ; q)} s_{k_{1} \ldots k_{j}}^{(j ; r)} s_{k_{1} \ldots k_{j}}^{(j ; ;)}=\left(g_{p q}^{(n ; j)} g_{r s}^{(n ; j)}\right)^{-1} T_{\lambda_{1} \ldots \lambda_{n}}^{(j ; p)} T_{\lambda_{1} \ldots \lambda_{n}}^{(j ; q)} S_{k_{1} \ldots k_{n}}^{(j ; r)} S_{k_{1} \ldots k_{n}}^{(j ; s)}$

which is valid provided no elements of the metric tensor $g^{(n ; j)}$ are zero. (In fact zeros only occur for $n>3$.) Inserting (2.19) into (2.18) gives the desired final result:

$$
\begin{aligned}
\left\langle\left|T_{i_{1} \ldots i_{n}} S_{i_{1} \ldots i_{n}}\right|^{2}\right\rangle & =\sum_{j=0}^{n}(2 j+1)^{-1} \sum_{p, q}^{N_{n}^{(n)}} \sum_{r, s}^{N_{n}^{\left(j^{\prime}\right)}}\left(\frac{g_{p r}^{(n ; j)}}{g_{p q}^{(n ; j)}} \frac{g_{q s}^{(n ; j)}}{g_{r s}^{(n ; j)}}\right) \\
& \times T_{\lambda_{1} \ldots \lambda_{n}}^{(j ; p)} \bar{T}_{\lambda_{1} \ldots \lambda_{n}}^{(j ; q)} S_{k_{1} \ldots k_{n}}^{(j ; r)} \bar{S}_{k_{1} \ldots k_{n}}^{(j ; s)} .
\end{aligned}
$$

Here the Greek indices indicate, as before, reference to a molecule-fixed frame in which the molecular response tensors are rotation invariant. Since the only matrix elements which are non-zero are those in which the weights of the molecular response tensors and the radiation field tensors are the same, the rotational averaging matrix takes on a block diagonal form. The rotational averaging matrix coefficients can readily be calculated with the aid of table 1 which gives the explicit form for $g_{p p^{\prime}}^{(n ; j)}$ up to rank

\begin{tabular}{|c|c|c|c|c|c|c|c|}
\hline Rank & Weight & \multicolumn{6}{|c|}{$g_{p p}^{(n ; j)}$} \\
\hline 2 & 2 & \multicolumn{6}{|c|}{1} \\
\hline 2 & 1 & \multicolumn{6}{|c|}{2} \\
\hline 2 & 0 & \multicolumn{6}{|c|}{3} \\
\hline 3 & 3 & \multicolumn{6}{|c|}{1} \\
\hline 3 & 2 & \multicolumn{6}{|c|}{$\left(\begin{array}{ll}2 & 1 \\
1 & 2\end{array}\right)$} \\
\hline 3 & 1 & \multicolumn{6}{|c|}{$\left(\begin{array}{lll}3 & 1 & 1 \\
1 & 3 & 1 \\
1 & 1 & 3\end{array}\right)$} \\
\hline 3 & 0 & \multicolumn{6}{|c|}{6} \\
\hline 4 & 4 & \multicolumn{6}{|c|}{1} \\
\hline 4 & 3 & & & $\begin{array}{l}2 \\
1 \\
1\end{array}$ & $\begin{array}{rr}1 & - \\
2 & - \\
-1 & \end{array}$ & $\left.\begin{array}{l}-1 \\
-1 \\
2\end{array}\right)$ & \\
\hline 4 & 2 & & $\left(\begin{array}{l}3 \\
1 \\
1 \\
1 \\
1 \\
0\end{array}\right.$ & $\begin{array}{l}1 \\
3 \\
1 \\
1 \\
0 \\
1\end{array}$ & $\begin{array}{l}1 \\
1 \\
0 \\
3 \\
1 \\
1\end{array}$ & $\begin{array}{ll}1 & 0 \\
0 & 1 \\
1 & 1 \\
1 & 1 \\
3 & 1 \\
1 & 3\end{array}$ & \\
\hline 4 & 1 & $\left(\begin{array}{r}6 \\
2 \\
2 \\
-2 \\
-2 \\
0\end{array}\right.$ & $\begin{array}{r}2 \\
6 \\
2 \\
2 \\
0 \\
-2\end{array}$ & $\begin{array}{l}2 \\
2 \\
6 \\
0 \\
2 \\
2\end{array}$ & $\begin{array}{r}-2 \\
2 \\
0 \\
6 \\
2 \\
-2\end{array}$ & $\begin{array}{r}-2 \\
0 \\
2 \\
2 \\
6 \\
2\end{array}$ & $\begin{array}{r}0 \\
-2 \\
2 \\
-2 \\
2 \\
6\end{array}$ \\
\hline 4 & 0 & & & $\left(\begin{array}{l}9 \\
3 \\
3\end{array}\right)$ & $\begin{array}{ll}3 & 3 \\
9 & 3 \\
3 & 9\end{array}$ & & \\
\hline
\end{tabular}
$4[11-13,15]$.

Table 1. Explicit form of the metric $g_{p p}^{(n ; j)}$ up to rank 4. 
As mentioned above, the result given in $(2.20)$ is correct so long as neither $g_{p q}^{(n ; j)}$ nor $g_{r s}^{(n ; j)}$ is zero. Although this condition is satisfied for $n \leqslant 3$, for $n=4$ the result is more complicated. In this case $g_{p q}^{(4 ; j)}$ are zero when $j=1,2$ and $q=N_{j}^{(n)}+1-p$. For these elements the following identity can be used to re-express (2.18) in an alternative embedded irreducible form:

$$
\begin{aligned}
t_{\lambda_{1} \ldots \lambda_{j}}^{(j ; p)} t_{\lambda_{1} \ldots \lambda_{j}}^{(j ; q)} s_{k_{1} \ldots k_{j}}^{(j ; r)} s_{k_{1} \ldots k_{j}}^{(j ; s)} \\
=\sum_{t, u} \tilde{g}_{(4 ; j)}^{p t} \tilde{g}_{(4 ; j)}^{r u} G_{\mu_{1} \ldots \mu_{4} ; \lambda_{1} \ldots \lambda_{j}}^{(0 ; t)} \tilde{G}_{\lambda_{1} \ldots \lambda_{j} ; \rho_{1} \ldots \rho_{4}}^{(0 ; q)} G_{k_{1} \ldots k_{4} ; i_{1} \ldots i_{j}}^{(0 ; u)} \\
\quad \times \tilde{G}_{i_{1} \ldots i_{j} ; l_{1} \ldots l_{4}}^{(0 ; s)} T_{\mu_{1} \ldots \mu_{4}}^{(j ; p)} T_{\rho_{1} \ldots \rho_{4}}^{(j ; ;)} S_{k_{1} \ldots k_{4}}^{(j ; r)} S_{l_{1} \ldots l_{4}}^{(j ; s)}
\end{aligned}
$$

where $g_{(4 ; j)}$ is the inverse metric defined by

$$
\sum_{q} \tilde{g}_{(4 ; j)}^{p q} g_{q r}^{(4 ; j)}=\delta_{r}^{p}
$$

However, the fact that the contributions $T_{\lambda_{1} \ldots \lambda_{4}}^{(j ; p)} \bar{T}_{\lambda_{1} \ldots \lambda_{4}^{j}}^{\left(j ; N^{(n)}+1-p\right)}$ vanish means that this average cannot be expressed in terms of a linear combination of the quadruply contracted tensors of the form $T_{\lambda_{1} \ldots \lambda_{4}}^{(j ; p)} \bar{T}_{\lambda_{1} \ldots \lambda_{4}}^{(j ; q)}$. This is verified by the fact that $G_{\mu_{1} \ldots \mu_{4} ; \lambda_{1} \ldots \lambda_{j}}^{\left(0 ; \lambda_{1}\right.} \tilde{G}_{\lambda_{1} \ldots \lambda_{j} ; \rho_{1} \ldots \rho_{4}}^{(0 ; q)}$ does not map the tensor product $T_{\mu_{1} \ldots \mu_{4}}^{(j ; p)} \bar{T}_{\rho_{1} \ldots \rho_{4}}^{\left(j ; N_{j}\left(N_{1}\right)+1-p\right)}$ into an irreducible result of the form $T_{\lambda_{1} \ldots \lambda_{4}}^{(j ; r)} \bar{T}_{\lambda_{1} \ldots \lambda_{4}}^{(j ; s)}$.

Explicit results for the rotational average of $(1.1)$ up to rank $6(n=3)$ are as follows.

Rank 2.

$$
\left\langle\left|T_{i} S_{i}\right|^{2}\right\rangle=\sum_{j=0}^{1}(2 j+1)^{-1} T_{\lambda}^{(j)} \bar{T}_{\lambda}^{(j)} S_{i}^{(j)} \bar{S}_{i}^{(j)}
$$

Rank 4.

$$
\left\langle\left|T_{i j} S_{i j}\right|^{2}\right\rangle=\sum_{j=0}^{2}(2 j+1)^{-1} T_{\lambda \mu}^{(j)} \bar{T}_{\lambda \mu}^{(j)} S_{i j}^{(j)} \bar{S}_{i j}^{(j)}
$$

Rank 6.

$$
\begin{aligned}
& \left\langle\left|T_{i j k} S_{i j k}\right|^{2}\right\rangle=T_{\lambda \mu \nu}^{(0)} \bar{T}_{\lambda \mu \nu}^{(0)} S_{i j k}^{(0)} \bar{S}_{i j k}^{(0)} \\
& \left.+\left(\begin{array}{ll}
T_{\lambda \mu \nu}^{(\alpha)} & \bar{T}_{\lambda \mu \nu}^{(1 \alpha)} \\
T_{\lambda \mu \nu}^{(1 \alpha)} & \bar{T}_{\lambda \mu \nu}^{(1 \beta)} \\
T_{\lambda \mu \nu}^{(1 \alpha)} & \bar{T}_{\lambda \mu \nu}^{(1 \gamma)} \\
T_{\lambda \mu \nu}^{(1 \beta)} & \bar{T}_{\lambda \mu \nu}^{(1 \alpha)} \\
T_{\lambda \mu \nu}^{(1 \beta)} & \bar{T}_{\lambda \mu \nu}^{(1 \beta)} \\
T_{\lambda \mu \nu}^{(1 \beta)} & \bar{T}_{\lambda \mu \nu}^{(1 \gamma)} \\
T_{\lambda \mu \nu}^{(1 \gamma)} & T_{\lambda \mu \nu}^{(1 \alpha)} \\
T_{\lambda \mu \nu}^{(1 \gamma)} & \bar{T}_{\lambda \mu \nu}^{(1 \beta)} \\
T_{\lambda \mu \nu}^{(1 \gamma)} & \bar{T}_{\lambda \mu \nu}^{(1 \gamma)}
\end{array}\right)^{\top}\right)^{\top} \frac{1}{48}\left(\begin{array}{rrrrrrrrr}
16 & 16 & 16 & 16 & 1 & -4 & 16 & -4 & 1 \\
16 & 256 & -64 & 16 & 16 & 16 & 16 & -64 & -4 \\
16 & -64 & 256 & 16 & -4 & -64 & 16 & 16 & 16 \\
16 & 16 & 16 & 256 & 16 & -64 & -64 & 16 & -4 \\
1 & 16 & -4 & 16 & 16 & 16 & -4 & 16 & 1 \\
-4 & 16 & -64 & -64 & 16 & 256 & 16 & 16 & 16 \\
16 & 16 & 16 & -64 & -4 & 16 & 256 & -64 & 16 \\
-4 & -64 & 16 & 16 & 16 & 16 & -64 & 256 & 16 \\
1 & -4 & 16 & -4 & 1 & 16 & 16 & 16 & 16
\end{array}\right)
\end{aligned}
$$




$$
\begin{aligned}
& \left(\begin{array}{ll}
S_{i j k}^{(1 \alpha)} & \bar{S}_{i j k}^{(1 \alpha)} \\
S_{i j k}^{(1 \alpha)} & \bar{S}_{i j k}^{(1 \beta)} \\
S_{i j k}^{(1 \alpha)} & \bar{S}_{i j k}^{(1 \gamma)} \\
S_{i j k}^{(1 \beta)} & \bar{S}_{i j k}^{(1 \alpha)} \\
S_{i j k}^{(1 \beta)} & \bar{S}_{i j k}^{(1 \beta)} \\
S_{i j k}^{(1 \beta)} & \bar{S}_{i j k}^{(1)} \\
S_{i j k}^{(1 \gamma)} & \bar{S}_{i j k}^{(1 \alpha)} \\
S_{i j k}^{(1 \gamma)} & S_{i j k}^{(1 \beta)} \\
S_{i j k}^{(1) \gamma} & \bar{S}_{i j k}^{(1 \gamma)}
\end{array}\right)+\frac{1}{20}\left(\begin{array}{ll}
T_{\lambda \mu \nu}^{(2 \alpha)} & \bar{T}_{\lambda \mu \nu}^{(2 \alpha)} \\
T_{\lambda \mu \nu}^{(2 \alpha)} & \bar{T}_{\lambda \mu \nu}^{(2 \beta)} \\
T_{\lambda \mu \nu}^{(2 \beta)} & \bar{T}_{\lambda \mu \nu}^{(2 \alpha)} \\
T_{\lambda \mu \nu}^{(2 \beta)} & \bar{T}_{\lambda \mu \nu}^{(2 \beta)}
\end{array}\right) \\
& +\frac{1}{7} T_{\lambda \mu \nu}^{(3)} \bar{T}_{\lambda \mu \nu}^{(3)} S_{i j k}^{(3)} \bar{S}_{i j k}^{(3)} .
\end{aligned}
$$

\section{Correspondence with conventional rotational averages}

It is possible to use the principles expounded above to generate earlier results obtained in reducible tensor form [2-8]. If we convert the natural tensors in (2.18) into their reducible forms in rank- $n$ space, through the use of the following inverse mapping formulae:

$$
\begin{aligned}
& t_{\lambda_{1} \ldots \lambda_{j}}^{(j ; p)}=\tilde{G}_{\lambda_{1} \ldots \lambda_{j} ; \mu_{1} \ldots \mu_{n}}^{(0 ; p)} T_{\mu_{1} \ldots \mu_{n}} \\
& \bar{t}_{\lambda_{1} \ldots \lambda_{j}}^{(j ; q)}=\tilde{G}_{\lambda_{1} \ldots \lambda_{j} ; \nu_{1} \ldots \nu_{n}}^{(0 ; q)} \bar{T}_{\nu_{1} \ldots \nu_{n}} \\
& s_{k_{1} \ldots k_{j}}^{(j ; r)}=\tilde{G}_{k_{1} \ldots k_{j} ; l_{1} \ldots l_{n}}^{(0 ; r)} S_{l_{1} \ldots l_{n}} \\
& \bar{s}_{k_{1} \ldots k_{j}}^{(j ; s)}=\tilde{G}_{k_{1} \ldots k_{j} ; m_{1} \ldots m_{n}}^{(0 ; s)} \bar{S}_{m_{1} \ldots m_{n}}
\end{aligned}
$$

we find that on substitution of (3.1)-(3.4) into (2.19) we obtain the necessary average expressed in terms of reducible tensor isomers, i.e.

$$
\begin{aligned}
\left\langle T_{i_{1} \ldots i_{n}} \bar{T}_{i_{n+1} \ldots i_{2 n}}\right. & \left.S_{i_{1} \ldots i_{n}} \bar{S}_{i_{n+1} \ldots i_{2 n}}\right\rangle \\
= & \sum_{j=0}(2 j+1)^{-1} \sum_{p, q} \sum_{r, s} g_{p q}^{(n ; j)} g_{r s}^{(n ; j)} \tilde{G}_{k_{1} \ldots k_{j} ; i_{1} \ldots l_{n}}^{(0 ; r)} \tilde{G}_{k_{1} \ldots k_{j} ; m_{1} \ldots m_{n}}^{(0 ; s)} \tilde{G}_{\lambda_{1} \ldots \lambda_{j} ; \mu_{1} \ldots \mu_{n}}^{(0 ; p)} \\
& \times \tilde{G}_{\lambda_{1} \ldots \lambda_{j} ; \nu_{1} \ldots \nu_{n}}^{(0 ; q)} T_{\mu_{1} \ldots \mu_{n}} \bar{T}_{\nu_{1} \ldots \nu_{n}} S_{l_{1} \ldots l_{n}} \bar{S}_{m_{1} \ldots m_{n}} .
\end{aligned}
$$

As an example, we derive the second-rank tensor average $(n=1)$. Using the information given in table 2 together with (3.5), and using the affine relation

$$
G_{k_{1} \ldots k_{n} ; l_{1} \ldots l_{n}}^{(0 ; p)} G_{k_{1} \ldots k_{n} ; m_{1} \ldots m_{n}}^{\left(0 ; m_{n}\right.}=\prod_{l_{1} \ldots l_{n} ; m_{1} \ldots m_{n}}^{(n ; n)}
$$

Table 2. Tensor mappings for a second-rank tensor.

\begin{tabular}{lll}
\hline$j$ & $g_{p q}^{(2 ; j)}$ & $G_{l_{1}, \ldots l_{i} ; k_{1} k_{2}}^{(0, p)}$ \\
\hline 2 & 1 & $\frac{1}{2}\left(\delta_{i_{1} k_{1}} \delta_{l_{2} k_{2}}+\delta_{l_{1} k_{2}} \delta_{l_{2} k_{1}}\right)-\frac{1}{3} \delta_{l_{1} l_{2}} \delta_{k_{1} k_{2}}$ \\
1 & 2 & $\frac{1}{2} \varepsilon_{l_{1} k_{1} k_{2}}$ \\
0 & 3 & $\frac{1}{3} \delta_{k_{1} k_{2}}$ \\
\hline
\end{tabular}


where the mapping on the right projects out the rank- $n$, weight- $n$, tensor representation from the reducible tensor subspace, we obtain the following result:

$$
\begin{aligned}
\left\langle\left|T_{i j} S_{i j}\right|\right\rangle^{2}=\left\{\frac{1}{5}[\right. & \left.\frac{1}{2}\left(\delta_{l_{1} k_{1}} \delta_{l_{2} k_{2}}+\delta_{l_{1} k_{2}} \delta_{l_{2} k_{1}}\right)-\frac{1}{3} \delta_{l_{1} l_{2}} \delta_{k_{1} k_{2}}\right]\left[\frac{1}{2}\left(\delta_{\mu_{1} \nu_{1}} \delta_{\mu_{2} \nu_{2}}+\delta_{\mu_{1} \nu_{2}} \delta_{\mu_{2} \nu_{1}}\right)-\frac{1}{3} \delta_{\mu_{1} \mu_{2}} \delta_{\nu_{1} \nu_{2}}\right] \\
& +\frac{4}{3}\left[\left(\frac{1}{2} \varepsilon_{l_{1} k_{1} k_{2}}\right)\left(\frac{1}{2} \varepsilon_{l_{1} m_{1} m_{2}}\right)\left(\frac{1}{2} \varepsilon_{\lambda_{1} \nu_{1} \nu_{2}}\right)\left(\frac{1}{2} \varepsilon_{\lambda_{1} \mu_{1} \mu_{2}}\right)\right] \\
& \left.+\frac{1}{9} \delta_{l_{1} l_{2}} \delta_{m_{1} m_{2}} \delta_{\mu_{1} \mu_{2}} \delta_{\nu_{1} \nu_{2}}\right\} T_{\mu_{1} \mu_{2}} \bar{T}_{\nu_{1} \nu_{2}} S_{l_{1} l_{2}} \bar{S}_{m_{1} m_{2}} .
\end{aligned}
$$

By executing the necessary index contractions we find that we can express the result as $\frac{1}{30}\left(\begin{array}{ll}\delta_{l_{1} l_{2}} & \delta_{m_{1} m_{2}} \\ \delta_{l_{1} m_{1}} & \delta_{l_{2} m_{2}} \\ \delta_{l_{1} m_{2}} & \delta_{l_{2} m_{1}}\end{array}\right)^{\mathrm{T}}\left(\begin{array}{rrr}4 & -1 & -1 \\ -1 & 4 & -1 \\ -1 & -1 & 4\end{array}\right)\left(\begin{array}{ll}\delta_{\mu_{1} \mu_{2}} & \delta_{\nu_{1} \nu_{2}} \\ \delta_{\mu_{1} \nu_{1}} & \delta_{\mu_{2} \nu_{2}} \\ \delta_{\mu_{1} \nu_{2}} & \delta_{\mu_{2} \nu_{1}}\end{array}\right) T_{\mu_{1} \mu_{2}} \bar{T}_{\nu_{1} \nu_{2}} S_{l_{1} l_{2}} \bar{S}_{m_{1} m_{2}}$

which is the more usual form in which the rotational average appears [7]. Results for higher-rank rotational averages can be obtained through the use of (3.5) and the necessary mapping formulae given by Coope et al [11-13]. Although this method affords no calculational advantages over over methods employed to evaluate isotropic tensor averages, it serves as a powerful illustration of the use of irreducible cartesian tensor calculus, and may additionally serve as a means of veryifying results.

\section{Rotational averages involving tensor products of odd rank}

Up to this point we have principally been concerned with the calculation of isotropic averages involving only the square of the leading term in the probability amplitude. However, there are certain chiral effects which arise in connection with linear and non-linear light scattering which require the evaluation of odd-rank cross terms $[16,17]$. Following a similar method one finds that such averages are given by

$$
\begin{aligned}
\left\langle S_{i_{1} \ldots i_{n}} S_{j_{1} \ldots j_{n+1}}^{\prime}\right. & \left.T_{i_{1} \ldots i_{n}} T_{j_{1} \ldots j_{n+1}}^{\prime}\right\rangle \\
= & \sum_{j=0}^{n} \sum_{p, q} \sum_{s^{\prime}, r^{\prime}}(2 j+1)^{-1} g_{p q}^{(n ; j)} g_{(n+1 ; j)}^{s^{\prime} r^{\prime}} \tilde{G}_{\lambda_{1} \ldots \lambda_{j} ; \mu_{1} \ldots \mu_{n}}^{(0 ; p)} \tilde{G}_{i_{1} \ldots i_{j} ; k_{1} \ldots k_{n}}^{(0 ; q)} \\
& \times G_{\rho_{1} \ldots \rho_{n+1} ; \lambda_{1} \ldots \lambda_{j}}^{\left(0 ; r^{\prime}\right)} G_{l_{1} \ldots l_{n+1} ; i_{1} \ldots i_{j}}^{(0 ; s)} T_{\mu_{1} \ldots \mu_{n}} T_{\rho_{1} \ldots \rho_{n+1}}^{\prime} S_{k_{1} \ldots k_{n}} S_{l_{1} \ldots l_{n+1}}^{\prime}
\end{aligned}
$$

which represents the main result in reducible form. The irreducible counterpart of equation (4.1) is found to be

$$
\begin{aligned}
\left\langle S_{i_{1} \ldots i_{n}} S_{j_{1} \ldots j_{n+1}}^{\prime}\right. & \left.T_{i_{1} \ldots i_{n}} T_{j_{1} \ldots j_{n+1}}^{\prime}\right\rangle \\
= & \sum_{j=0}^{n} \sum_{p, q, r, s} \sum_{s^{\prime}, r^{\prime}}(2 j+1)^{-1} g_{p q}^{(n ; j)} g_{(n+1 ; j)}^{r r^{\prime}} g_{(n+1 ; j)}^{s s^{\prime}} g_{r s}^{(n ; j)} \\
& \times \tilde{G}_{\lambda_{1} \ldots \lambda_{j} ; \mu_{1} \ldots \mu_{n}}^{(0 ; ;)} \tilde{G}_{i_{1} \ldots i_{j} ; k_{1} \ldots k_{n}}^{(0 ; q)} G_{\rho_{1} \ldots \rho_{n+1} ; \lambda_{1} \ldots \lambda_{j}}^{\left(0 ; r^{\prime}\right)} G_{l_{1} \ldots i_{n+1} ; i_{1} \ldots i_{j}}^{\left(0 ; s^{\prime}\right)} \\
& \times T_{\mu_{1} \ldots \mu_{n}}^{(j ; q)} T_{\rho_{1} \ldots \rho_{n+1}}^{(j ; s)} S_{k_{1} \ldots k_{n}}^{(j ; p)} S_{l_{1} \ldots l_{n+1} \cdot}^{(j ; ;)}
\end{aligned}
$$

The rank-3 $(n=1)$ and the rank-5 $(n=2)$ rotational averages have the following explicit forms.

$n=1$.

$$
\begin{aligned}
\left\langle S_{i_{1}} S_{j_{1} j_{2}}^{\prime} T_{i_{1}} T_{j_{1} j_{2}}^{\prime}\right\rangle & =\frac{1}{6} \varepsilon_{\mu_{1} \mu_{2} \mu_{3}} \varepsilon_{l_{1} l_{2} l_{3}} T_{\mu_{1}} T_{\mu_{2} \mu_{3}}^{\prime} S_{l_{1}} S_{l_{2} l_{3}}^{\prime} \\
& =\frac{1}{6} \varepsilon_{l_{1} l_{2} l_{3}} \varepsilon_{\mu_{1} \mu_{2} \mu_{3}} S_{l_{1}}^{(1)} S_{l_{2} l_{3}}^{(1)} T_{\mu_{1}}^{(1)} T_{\mu_{2} \mu_{3}}^{(1)} .
\end{aligned}
$$


$n=2$.

$$
\begin{aligned}
& \left\langle S_{i_{1} i_{2}} S_{j_{1} j_{2} j_{3}}^{\prime} T_{i_{1} i_{2}} T_{j_{1} j_{2} j_{3}}^{\prime}\right\rangle \\
& =\frac{1}{30}\left(\begin{array}{ll}
\varepsilon_{\iota_{1} \iota_{2} t_{3}} & \delta_{\iota_{4} \iota_{5}} \\
\varepsilon_{t_{1} \iota_{2} \iota_{4}} & \delta_{t_{3} \iota_{5}} \\
\varepsilon_{\iota_{1} \iota_{2} \iota_{5}} & \delta_{\iota_{3} \iota_{4}} \\
\varepsilon_{\iota_{1} \iota_{3} \iota_{4}} & \delta_{\iota_{2} \iota_{5}} \\
\varepsilon_{\iota_{1} \iota_{3} \iota_{5}} & \delta_{t_{2} \iota_{4}} \\
\varepsilon_{\iota_{1} \iota_{4} \iota_{5}} & \delta_{\iota_{2} \iota_{3}}
\end{array}\right)^{\top}\left(\begin{array}{rrrrrr}
3 & -1 & -1 & 1 & 1 & 0 \\
-1 & 3 & -1 & -1 & 0 & 1 \\
-1 & -1 & 3 & 0 & -1 & -1 \\
1 & -1 & 0 & 3 & -1 & 1 \\
1 & 0 & -1 & -1 & 3 & -1 \\
0 & 1 & -1 & 1 & -1 & 3
\end{array}\right)\left(\begin{array}{lll}
\varepsilon_{\lambda_{1} \lambda_{2} \lambda_{3}} & \delta_{\lambda_{4} \lambda_{5}} \\
\varepsilon_{\lambda_{1} \lambda_{2} \lambda_{4}} & \delta_{\lambda_{3} \lambda_{5}} \\
\varepsilon_{\lambda_{1} \lambda_{2} \lambda_{5}} & \delta_{\lambda_{3} \lambda_{4}} \\
\varepsilon_{\lambda_{1} \lambda_{3} \lambda_{4}} & \delta_{\lambda_{2} \lambda_{5}} \\
\varepsilon_{\lambda_{1} \lambda_{3} \lambda_{5}} & \delta_{\lambda_{2} \lambda_{4}} \\
\varepsilon_{\lambda_{1} \lambda_{4} \lambda_{5}} & \delta_{\lambda_{2} \lambda_{3}}
\end{array}\right) \\
& \times T_{\lambda_{4} \lambda_{5}} T_{\lambda_{1} \lambda_{2} \lambda_{3}}^{\prime} S_{\iota_{4} \iota_{5}} S_{\iota_{1} \iota_{2} \iota_{3}}^{\prime} \\
& =\frac{1}{18} \varepsilon_{l_{1} l_{2} l_{3}} S_{l_{4} l_{4}}^{(0)} S_{l_{1} l_{2} l_{3}}^{(0)} \varepsilon_{\mu_{1} \mu_{2} \mu_{3}} T_{\mu_{4} \mu_{4}}^{(0)} T_{\mu_{1} \mu_{2} \mu_{3}}^{(0)} \\
& +\frac{1}{600} S_{l_{4} l_{5}}^{(1)} T_{\mu_{4} \mu_{5}}^{(1)}\left(\begin{array}{ll}
\varepsilon_{\mu_{1} \mu_{4} \mu_{5}} & \delta_{\mu_{2} \mu_{3}} \\
\varepsilon_{\mu_{2} \mu_{4} \mu_{5}} & \delta_{\mu_{1} \mu_{3}} \\
\varepsilon_{\mu_{3} \mu_{4} \mu_{5}} & \delta_{\mu_{1} \mu_{2}}
\end{array}\right)^{\mathrm{T}}\left(\begin{array}{ccc}
4 T_{\mu_{1} \mu_{2} \mu_{3}}^{(1 ; 1)} & -T_{\mu_{1} \mu_{2} \mu_{3}}^{(1 ; 1)} & -T_{\mu_{1} \mu_{2} \mu_{3}}^{(1 ; 1)} \\
-T_{\mu_{1} \mu_{2} \mu_{3}}^{(1 ; 2)} & 4 T_{\mu_{1} \mu_{2} \mu_{3}}^{(1 ; 2)} & -T_{\mu_{1} \mu_{2} \mu_{3}}^{(1,2)} \\
-T_{\mu_{1} \mu_{2} \mu_{3}}^{(1 ; 3)} & -T_{\mu_{1} \mu_{2} \mu_{3}}^{(1 ; 3)} & 4 T_{\mu_{1} \mu_{2} \mu_{3}}^{(1 ; 3)}
\end{array}\right)
\end{aligned}
$$

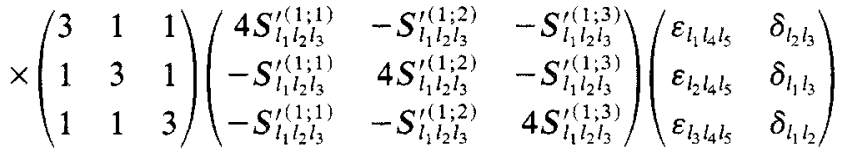

$$
\begin{aligned}
& +\frac{1}{45} S_{l_{4} l_{5}}^{(2)} T_{\mu_{4} \mu_{5}}^{(2)}\left(\begin{array}{ll}
E_{\left(\mu_{4} \mu_{5} ; \tau \mu_{1}\right)}^{(2)} & \varepsilon_{\tau \mu_{2} \mu_{3}} \\
E_{\left(\mu_{4} \mu_{5} ; \tau \mu_{2}\right)}^{(2)} & \varepsilon_{\tau \mu_{1} \mu_{3}}
\end{array}\right)^{\mathrm{T}}\left(\begin{array}{cc}
2 T_{\mu_{1} \mu_{2} \mu_{3}}^{(2 ; 1)} & -T_{\mu_{1}}^{\left(2 ; \mu_{2} \mu_{3}\right.} \\
-T_{\mu_{1} \mu_{2} \mu_{3}}^{(2 ; 2)} & 2 T_{\mu_{1} \mu_{2} \mu_{3}}^{(2 ; 2)}
\end{array}\right) \\
& \times\left(\begin{array}{ll}
2 & 1 \\
1 & 2
\end{array}\right)\left(\begin{array}{cc}
2 S_{l_{1} l_{2} l_{3}}^{(2 ; 1)} & -S_{l_{1}}^{\left(2 ; 2 l_{3}\right.} \\
-S_{l_{1}}^{\prime\left(2 ; l_{2} ; 1\right)} & 2 S_{l_{1} l_{2}}^{\prime\left(2 l_{2} l_{3}\right.}
\end{array}\right)\left(\begin{array}{cc}
E_{\left(l_{4} l_{5} ; l_{1}\right)}^{(2)} & \varepsilon_{t l_{2} l_{3}} \\
E_{\left(l_{4} l_{5} ; l_{2}\right)}^{(2)} & \varepsilon_{t l_{1} l_{3}}
\end{array}\right) .
\end{aligned}
$$

\section{Derivation of embedded irreducible isotropic averages from reducible isotropic averages}

It is possible to verify the results of $\S 2$ by an alternative method using a matrix formalism outlined in part by Andrews and Wilkes [18]. This method expresses each scalar $T_{\lambda_{1} \ldots \lambda_{n}}^{(j ; p)} \bar{T}_{\lambda_{1} \ldots \lambda_{n}}^{(j ; q)}$ in terms of the $Q_{n}$ linearly independent isotropic tensor isomers. First, we write the isotropic average as

$$
I_{i_{1} \ldots i_{2 n} ; \lambda_{1} \ldots \lambda_{2 n}}^{(2 n)}=\sum_{p, q} m_{p q}^{(2 n)} f_{i_{1} \ldots i_{2 n}}^{(2 n ; p)} g_{\lambda_{1} \ldots \lambda_{2 n}}^{(2 n ; q)}
$$

where $m_{p q}$ are numerical coefficients and $f^{(2 n ; p)}, g^{(2 n ; q)}$ denote the rank-2n isotropic tensors in the laboratory and molecular frames respectively. Writing

$$
t_{q}=g_{\lambda_{1} \ldots \lambda_{2 n}}^{(2 n ; q)} T_{\lambda_{1} \ldots \lambda_{n}} \bar{T}_{\lambda_{n+1} \ldots \lambda_{2 n}}
$$

and

$$
s_{p}=f_{i_{1} \ldots i_{2 n}}^{(2 n ; p)} S_{i_{1} \ldots i_{n}} \bar{S}_{i_{n+1} \ldots i_{2 n}}
$$

it is possible to write the rank- $2 n$ average in the form

$$
\left\langle S_{i_{1} \ldots i_{n}} \bar{S}_{i_{n+1} \ldots i_{2 n}} T_{i_{1} \ldots i_{n}} \bar{T}_{i_{n+1} \ldots i_{2 n}}\right\rangle=\sum_{p, q} m_{p q}^{(2 n)} s_{p} t_{q}
$$


Both the molecular parameters $t_{q}$ and the polarisation parameters $s_{p}$ can be reexpressed in terms of irreducible tensor products $t_{r}^{i}$ and $s_{s}^{i}$ by use of (2.3)-(2.6), leading to relations of the form

$$
t_{r}^{i}=\sum_{q=1}^{Q_{n}} j_{r q} t_{q}
$$

and

$$
t_{q}=\sum_{r=1}^{Q_{n}}\left(j^{-1}\right)_{q r} t_{r}^{i}
$$

where $j$ and $j^{-1}$ constitute index-symmetric numerical matrices of rank $Q_{n}$. It is thus possible to express the rotational average defined in (5.4) as

$$
\left\langle S_{i_{1} \ldots i_{n}} \bar{S}_{i_{n+1} \ldots i_{2 n}} T_{i_{1} \ldots i_{n}} \bar{T}_{i_{n+1} \ldots i_{2 n}}\right\rangle=\sum_{r, s} \sum_{p, q}\left(j^{-1}\right)_{p s} m_{p q}^{(2 n)}\left(j^{-1}\right)_{q r} s_{s}^{i} t_{r}^{i} .
$$

For ranks $n=2$ and $n=4$, averaging the result is trivial since there is only one representation for each weight, and consequently $\left(j^{-1}\right)_{p s}$ and $\left(j^{-1}\right)_{q r}$ are elements of $2 \times 2$ and $3 \times 3$ matrices. This is not the case for rank- 6 averaging, however, where $\left(j^{-1}\right)_{p s}$ and $\left(j^{-1}\right)_{q r}$ are elements of a $15 \times 15$ matrix. These elements have previously been calculated by Andrews and Wilkes [16].

As an example we again concentrate on the rank-4 average. From (5.5) and appendix 2 we have

$$
\left(\begin{array}{ll}
T_{\lambda \mu}^{(0)} & \bar{T}_{\lambda \mu}^{(0)} \\
T_{\lambda \mu}^{(1)} & \bar{T}_{\lambda \mu}^{(1)} \\
T_{\lambda \mu}^{(2)} & \bar{T}_{\lambda \mu}^{(2)}
\end{array}\right)=\frac{1}{6}\left(\begin{array}{rrr}
2 & 0 & 0 \\
0 & 3 & -3 \\
-2 & 3 & 3
\end{array}\right)\left(\begin{array}{ll}
T_{\nu \nu} & \bar{T}_{\tau \tau} \\
T_{\lambda \mu} & \bar{T}_{\lambda \mu} \\
T_{\lambda \mu} & \bar{T}_{\mu \lambda}
\end{array}\right)
$$

The inverse relation can be expressed in the form of (5.6) as follows:

$$
\left(\begin{array}{rrr}
3 & 0 & 0 \\
1 & 1 & 1 \\
1 & -1 & 1
\end{array}\right)\left(\begin{array}{ll}
T_{\lambda \mu}^{(0)} & \bar{T}_{\lambda \mu}^{(0)} \\
T_{\lambda \mu}^{(1)} & \bar{T}_{\lambda \mu}^{(1)} \\
T_{\lambda \mu}^{(2)} & \bar{T}_{\lambda \mu}^{(2)}
\end{array}\right)=\left(\begin{array}{ll}
T_{\nu \nu} & \bar{T}_{\tau \tau} \\
T_{\lambda \mu} & \bar{T}_{\lambda \mu} \\
T_{\lambda \mu} & \bar{T}_{\mu \lambda}
\end{array}\right)
$$

Inserting this relation into (5.7) gives the isotropic average as

$$
\frac{1}{30}\left(\begin{array}{ll}
S_{i j}^{(0)} & \bar{S}_{i j}^{(0)} \\
S_{i j}^{(1)} & \bar{S}_{i j}^{(1)} \\
S_{i j}^{(2)} & \bar{S}_{i j}^{(2)}
\end{array}\right)^{\mathrm{T}}\left(\begin{array}{rrr}
3 & 1 & 1 \\
0 & 1 & -1 \\
0 & 1 & 1
\end{array}\right)\left(\begin{array}{rrr}
4 & -1 & -1 \\
-1 & 4 & -1 \\
-1 & -1 & 4
\end{array}\right)\left(\begin{array}{rrr}
3 & 0 & 0 \\
1 & 1 & 1 \\
1 & -1 & 1
\end{array}\right)\left(\begin{array}{ll}
T_{\lambda \mu}^{(0)} & \bar{T}_{\lambda \mu}^{(0)} \\
T_{\lambda \mu}^{(1)} & \bar{T}_{\lambda \mu}^{(1)} \\
T_{\lambda \mu}^{(2)} & \bar{T}_{\lambda \mu}^{(2)}
\end{array}\right)
$$

which reduces to the earlier result

$$
\left\langle\left|T_{i j} S_{i j}\right|^{2}\right\rangle=\sum_{j=0}^{2}(2 j+1)^{-1} T_{\lambda \mu}^{(j)} \bar{T}_{\lambda \mu}^{(j)} S_{i j}^{(j)} \bar{S}_{i j}^{(j)} .
$$

\section{Conclusion}

In this paper we have shown how the principles of irreducible cartesian tensor analysis can be used to derive rotational averages for the observables associated with molecular processes described in terms of the Fermi golden rule. It has been demonstrated that the results are considerably simplified by expressing observables directly in terms of 
irreducible tensors, thus bringing the rotational averaging matrix into block diagonal form. We also have shown how the principles of cartesian tensor analysis can be used to derive the isotropic averages in a reducible form. This has served to verify results published in earlier papers, and to facilitate future extension to isotropic averages for any higher rank. Although the results given in this paper apply to three-dimensional rotational averaging, it would be relatively simple to adapt these results for twodimensional systems by a suitable adaptation of the relevant metric tensors.

\section{Acknowledgment}

Nick Blake gratefully acknowledges financial support from the Science and Engineering Research Council.

\section{Appendix 1. Proof of an identity}

$$
\begin{aligned}
T_{\lambda_{1} \ldots \lambda_{n}}^{(j ; p)} \bar{T}_{\lambda_{1} \ldots \lambda_{n}}^{(j ; q)} & S_{i_{1} \ldots i_{n}}^{(j ; r)} \bar{S}_{i_{1} \ldots i_{n}}^{(j ; s)} \\
= & G_{\lambda_{1} \ldots \lambda_{n} ; \rho_{1} \ldots \rho_{j}}^{(0 ; p)} G_{\lambda_{1} \ldots \lambda_{n} ; \sigma_{1} \ldots \sigma_{j}}^{(0 ; q)} G_{i_{1} \ldots i_{n} ; k_{1} \ldots k_{j}}^{(0 ; r)} G_{i_{1} \ldots i_{n} ; l_{1} \ldots l_{j}}^{(0 ; s)} \\
& \times t_{\rho_{1} \ldots \rho_{j}}^{(j ; p)} \bar{t}_{\sigma_{1} \ldots \sigma_{j}}^{(j ; q)} s_{k_{1} \ldots k_{j}}^{(j ; r)} \bar{s}_{l_{1} \ldots l_{j}}^{(j ; s)} .
\end{aligned}
$$

Using the inverse mappings

$$
\begin{aligned}
& G_{i_{1} \ldots i_{n} ; k_{1} \ldots k_{j}}^{(0 ; r)}=\sum_{r^{\prime}} g_{r r^{\prime}}^{(n ; j)} \tilde{G}_{k_{1} \ldots k_{j} ; i_{1} \ldots i_{n}}^{\left(0 ; r^{\prime}\right)} \\
& G_{\lambda_{1} \ldots \lambda_{n} ; \rho_{1} \ldots \rho_{j}}^{(0 ; p)}=\sum_{p^{\prime}} g_{p p^{\prime}}^{(n ; j)} \tilde{G}_{\rho_{1} \ldots \rho_{j} ; \lambda_{1} \ldots \lambda_{n}}^{\left(0 ; p^{\prime}\right)}
\end{aligned}
$$

and the identities

$$
\begin{aligned}
& \tilde{G}_{k_{1} \ldots k_{j} ; i_{1} \ldots i_{n}}^{\left(0 ; r^{\prime}\right.} G_{i_{1} \ldots i_{n} ; l_{1} \ldots l_{j}}^{(0 ; s)}=\delta_{r^{\prime} s} E_{k_{1} \ldots k_{j} ; l_{1} \ldots l_{j}}^{(j)} \\
& \tilde{G}_{\rho_{1} \ldots \rho_{j} ; \lambda_{1} \ldots \lambda_{n}}^{\left(0 ; p^{\prime}\right)} G_{\lambda_{1} \ldots \lambda_{n} ; \sigma_{1} \ldots \sigma_{j}}^{(0 ; q)}=\delta_{p^{\prime} q} E_{\rho_{1} \ldots \rho_{j} ; \sigma_{1} \ldots \sigma_{j}}^{(j)}
\end{aligned}
$$

we obtain from (A1)

$$
T_{\lambda_{1} \ldots \lambda_{n}}^{(j ; p)} \bar{T}_{\lambda_{1} \ldots \lambda_{n}}^{(j ; q)} S_{i_{1} \ldots i_{n}}^{(j ; r)} \bar{S}_{i_{1} \ldots i_{n}}^{(j ; s)}=g_{p q}^{(n ; j)} g_{r s}^{(n ; j)} s_{k_{1} \ldots k_{j}}^{(j ; r)} \bar{s}_{k_{1} \ldots k_{j}}^{(j ; r)} t_{\rho_{1} \ldots \rho_{j}}^{(j ; p)} \bar{t}_{\rho_{1} \ldots \rho_{j}}^{(j ; q)}
$$

or

$$
\left(g_{p q}^{(n ; j)} g_{r s}^{(n ; j)}\right)^{-1} T_{\lambda_{1} \ldots \lambda_{n}}^{(j ; p)} \bar{T}_{\lambda_{1} \ldots \lambda_{n}}^{(j ; q)} S_{i_{1} \ldots i_{n}}^{(j ; r)} \bar{S}_{i_{1} \ldots i_{n}}^{(j ; s)}=s_{k_{1} \ldots k_{j}}^{(j ; r)} \bar{s}_{k_{1} \ldots k_{j}}^{(j ; s)} t_{\rho_{1} \ldots \rho_{j}}^{(j ; p)} \bar{t}_{\rho_{1} \ldots \rho_{j}}^{(j ; q)}
$$

This is the identity used in (2.20).

Appendix 2. Explicit forms of the rank- $n$, weight- $j$, irreducible tensors $T_{s_{1} \ldots s_{n}}^{(j ; p)}$

Rank 2.

$$
\begin{aligned}
& T_{s_{1} s_{2}}^{(0)}=\frac{1}{3} \delta_{s_{1} s_{2}} T_{s_{p} s_{p}} \\
& T_{s_{1} s_{2}}^{(1)}=\frac{1}{2}\left(T_{s_{1} s_{2}}-T_{s_{2} s_{1}}\right) \\
& T_{s_{1} s_{2}}^{(2)}=\frac{1}{2}\left(T_{s_{1} s_{2}}+T_{s_{2} s_{1}}\right)-\frac{1}{2} \delta_{s_{1} s_{2}} T_{s_{p} s_{p}}
\end{aligned}
$$




\section{Rank 3.}

$$
\begin{aligned}
& T_{s_{1} s_{2} s_{3}}^{(0)}=\frac{1}{6} \varepsilon_{s_{1} s_{2} s_{3}} \varepsilon_{s_{p} s_{\sigma} s_{\tau}} T_{s_{p} s_{\sigma} s_{\tau}} \\
& T_{s_{1} s_{2} s_{3}}^{(1 ; 1)}=\frac{1}{10}\left(4 \delta_{s_{1} s_{2}} T_{s_{p} s_{p} s_{3}}-\delta_{s_{1} s_{3}} T_{s_{p} s_{p} s_{2}}-\delta_{s_{2} s_{3}} T_{s_{p} s_{p} s_{1}}\right) \\
& T_{s_{1} s_{2} s_{3}}^{(1 ; 2)}=\frac{1}{10}\left(-\delta_{s_{1} s_{2}} T_{s_{p} s_{3} s_{p}}+4 \delta_{s_{1} s_{3}} T_{s_{p} s_{2} s_{p}}-\delta_{s_{2} s_{3}} T_{s_{p} s_{1} s_{p}}\right) \\
& T_{s_{1} s_{2} s_{3}}^{(1 ; 3)}=\frac{1}{10}\left(-\delta_{s_{1} s_{2}} T_{s_{3} s_{p} s_{p}}-\delta_{s_{1} s_{3}} T_{s_{2} s_{p} s_{p}}+4 \delta_{s_{2} s_{3}} T_{s_{1} s_{p} s_{p}}\right) \\
& T_{s_{1} s_{2} s_{3}}^{(2 ; 1)}=\frac{1}{6} \varepsilon_{s_{1} s_{2} s_{\tau}}\left(2 \varepsilon_{s_{p} s_{\sigma} s_{\tau}} T_{s_{p} s_{\sigma} s_{3}}+2 \varepsilon_{s_{p} s_{\sigma} s_{3}} T_{s_{p} s_{\sigma} s_{\tau}}+\varepsilon_{s_{p} s_{\sigma} s_{\tau}} T_{s_{3} s_{p} s_{\sigma}}+\varepsilon_{s_{p} s_{\sigma} s_{3}} T_{s_{\tau} s_{\rho} s_{\sigma}}\right. \\
& \left.-2 \delta_{s_{1} s_{\tau}} \varepsilon_{s_{\pi} s_{p} s_{\sigma}} T_{s_{\pi} s_{\eta} s_{\sigma}}\right) \\
& T_{s_{1} s_{2} s_{3}}^{(2 ; 2)}=\frac{1}{6} \varepsilon_{s_{2} s_{3} s_{\tau}}\left(2 \varepsilon_{s_{p} s_{\sigma} s_{\tau}} T_{s_{1} s_{p} s_{\sigma}}+2 \varepsilon_{s_{p} s_{\sigma} s_{1}} T_{s_{\tau} s_{p} s_{\sigma}}+\varepsilon_{s_{p} s_{\sigma} s_{\tau}} T_{s_{p} s_{\sigma} s_{1}}+\varepsilon_{s_{p} s_{\sigma} s_{1}} T_{s_{p} s_{\sigma} s_{\tau}}\right. \\
& \left.-2 \delta_{s_{1} s_{\tau}} \varepsilon_{s_{\pi} s_{p} s_{\sigma}} T_{s_{\pi} s_{p} s_{\sigma}}\right) \\
& T_{s_{1} s_{2} s_{3}}^{(3)}=\frac{1}{6}\left(T_{s_{1} s_{2} s_{3}}+T_{s_{1} s_{3} s_{2}}+T_{s_{2} s_{1} s_{3}}+T_{s_{2} s_{3} s_{1}}+T_{s_{3} s_{1} s_{2}}+T_{s_{3} s_{2} s_{1}}\right) \\
& -\frac{1}{15}\left[\delta_{s_{1} s_{1}}\left(T_{s_{p} s_{p} s_{3}}+T_{s_{p} s_{3} s_{p}}+T_{s_{3} s_{p} s_{p}}\right)\right. \\
& \left.+\delta_{s_{1} s_{3}}\left(T_{s_{p} s_{p} s_{2}}+T_{s_{p} s_{2} s_{p}}+T_{s_{2} s_{p} s_{p}}\right)+\delta_{s_{2} s_{3}}\left(T_{s_{p} s_{p} s_{1}}+T_{s_{p} s_{1} s_{p}}+T_{s_{1} s_{p} s_{p}}\right)\right] \text {. }
\end{aligned}
$$

\section{References}

[1] Craig D P and Thirunamachandran T 1984 Molecular Quantum Electrodynamics-An Introduction to Molecule-Radiation Interactions (New York: Academic)

[2] Kielich S 1961 Acta Phys. Polon. 20433

[3] Monson P R and McClain W M 1970 J. Chem. Phys. 5329

[4] McClain W M 1972 J. Chem. Phys, 572264

[5] Healy W P 1974 J. Phys. B: At. Mol. Phys. 71633

[6] Power E A and Thirunamachandran T 1974 J. Chem. Phys. 603695

[7] Andrews D L and Thirunamachandran T 1977 J. Chem. Phys. 675026

[8] Wagnière G $1982 \mathrm{~J}$. Chem. Phys. 76473

[9] McClain W M, Tian D and Ghoul W A 1987 J. Chem. Phys. 874986

[10] Andrews D L and Ghoul W A $1981 J$. Phys. A: Math. Gen. 141281

[11] Coope J A R, Snider R F and McCourt F R 1965 J. Chem. Phys. 432269

[12] Coope J A R and Snider R F 1970 J. Math. Phys. 11993

[13] Coope J A R 1970 J. Math. Phys. 111591

[14] Mikhailov V V 1977 J. Phys. A: Math. Gen. 10147

[15] Andrews D L and Ghoul W A 1982 Phys. Rev. A 252647

[16] Andrews D L and Thirunamachandran T 1979 J. Chem. Phys. 701027

[17] Andrews D L 1980 J. Chem. Phys. 724141

[18] Andrews D L and Wilkes P J 1985 J. Chem. Phys. 832009 